\title{
Biocompatible Quantum Dot Nanocolloids Stabilized by Gum Arabic
}

\author{
Chansik Park, Kook Hec Lim, Dongwook Kwon, and Tae Hyun Yoon ${ }^{\star}$
}

\author{
Laboratory of Nanoscale Characterization and Environmental Chemistry. Department of Chemistry, Ianyang lniversity; \\ Seoul 133-79/. Korea. "E-mail: tueyooniahamang.ackr \\ Received March 12, 2008
}

Key Words : Quantum dots, Gum arabic, Photostability, Nanocolloids

Quantum dots (QDs) are semiconductor nanocrystals with size-dependent optical properties, which have great potential in many areas of application, such as solar cells, light-emitting devices, biological and medical imaging.' Of particular importance, they are considered as a new type of fluorescence probe for multicolor imaging due to their narrow emission spectra, high quantum yield and single excitation light source requirement for multiple light emission. ${ }^{2}$ Therefore, preparation of well-dispersed and photochemicallystable QD nanocolloids in aqueous solution is prerequisite for many applications and numerous methods have been developed for this purpose..$^{2,3}$ However, these surface modification processes often involve covalent bond formation between ligand and QD surface and cause significant changes in the physicochemical states of the QD surface atoms. As a result, dramatic degradation in the desired properties of QDs were frequently observed, such as significant decreases in photochemical and/or colloidal stability, quantum yields $(Q Y)$ and acceleration of coagulation/precipitation process. ${ }^{4}$

Io minimize these deteriorations in the desired properties of QDs, alternative methods have been developed. For instance, several QD surface modification methods based on hydrophobic interaction of surfactants, ${ }^{5}$ synthetic block copolymers $^{6}$ and phospholipid ${ }^{?}$ with capping molecules (e.g. Trioctylphosphine oxide ( $\left.\mathrm{TOP}^{\circ} \mathrm{O}\right)$ ) of the QD surfaces were recently reported. In this short note, we will describe a simple but very effective method for the preparation of biocompatible QD nanocolloids in water, via nonspecific physical interaction with natural polysaccharides (i.e., Gum Arabic), which also induced significant enhancements in the desired properties for biological and medical imaging. such as increased quantum yields and photochemical stabilities.

Gum Arabic (GA) is a highly branched, neutral or slightly acidic arabinogalactan polysaccharides, obtained naturally from the stems and branches of Acacia Senegal tree. ${ }^{8}$ According to recent characterization studies, ${ }^{9}$ it is composed of three main components: arabinogalactan-peptide, arabinogalactan-protein and glycoproteins. These nontoxic and biocompatible properties of GA made it widely used in food and pharmaceutical industry as additives or emulsifying agent. ${ }^{10}$ Moreover, it is also being increasingly used as a stabilizer for various novel nanomaterials, such as carbon nanotubes, ${ }^{11}$ nano-gold, ${ }^{12}$ nano-silver ${ }^{13}$ and oxide nanoparticles. ${ }^{14.15}$ However, as far as we know, no application of $\mathrm{GA}$ for the stabilization of $\mathrm{QD}$ nanoparticles has been reported yet and this is the first application of GA, a naturally occurring polymer with superb dispersing ability, for the preparation of QD nanocolloids.

Using the simple method described in experimental section, which is also previously suggested and used by Fan et $a l^{5}$ for the preparation of surfactant assisted QD micelle. ${ }^{\text {GNVTOPOO } Q D}$ nanocolloid was prepared. As-prepared GATOPO $Q D$ solution have very clear reddish orange color with typical UV/Vis absorption and fluorescence spectra of QDs ( $1^{\text {st }}$ exciton absorption peak at $564 \mathrm{~nm}$ and emission peak at $576 \mathrm{~nm}$, Figure 1(a)). Hydrodynamic size of (inTCPY $Q D$ measured by dynamic light scattering (DLS) method was $25.5 \mathrm{~nm}$, which is larger than those of ${ }^{\mathrm{MPA}} \mathrm{QD}(4.6 \mathrm{~nm})$ and (TAB:TOPCOD $(20.7 \mathrm{~nm})$. This increased hydrodynamic size of ${ }^{(\mathrm{CATOPO}} \mathrm{QD}$ nanocolloid is probably due to the much complex networked structures of GA. Based on IUPAC method. ${ }^{19}$ which use absorption and fluorescence peak areas of QDs relative to Rhodamine $6 \mathrm{G}(\mathrm{QY}=0.99)$, relative Quantum Yields $(Q Y)$ were also estimated. Compared to the $Q Y$ of

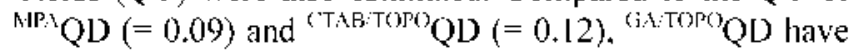
shown significant improvement in its QY $(=0.27)$.

The photochemical stability of ${ }^{\text {(IATOPO }}$ QD were also tested under UV exposure conditions and presented in Figure 2. As
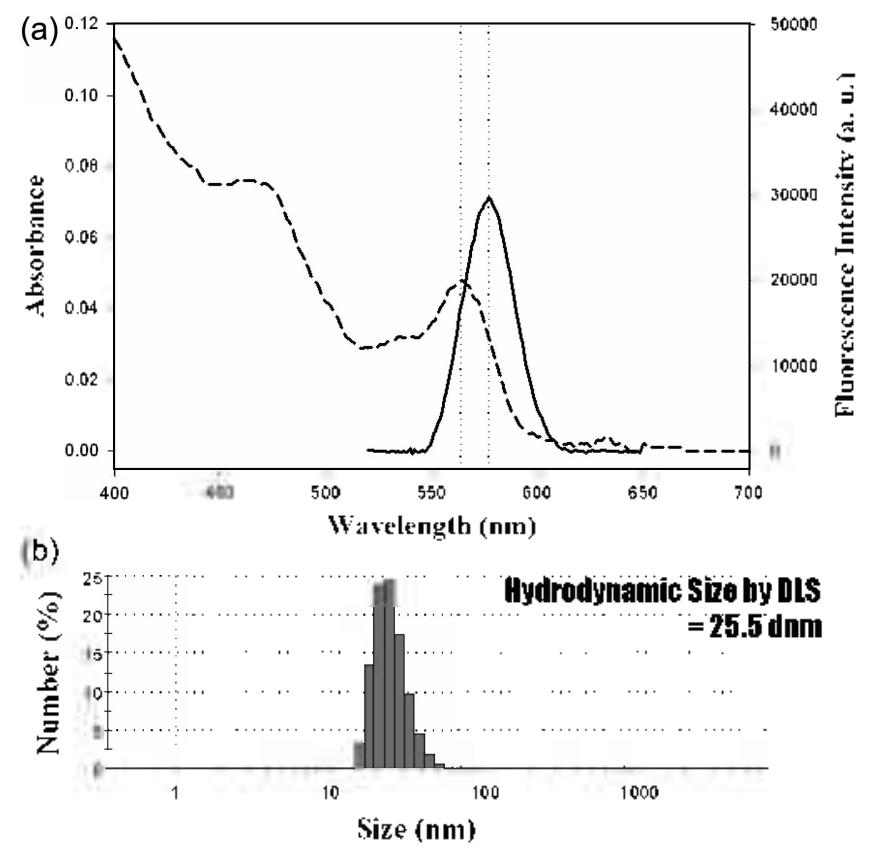

Figure 1. (a) LIV/vis absorption and fluorescence spectra and (b) hydrodynamic size of ${ }^{(i \mathrm{i})(\mathrm{T})(0)} \mathrm{QDS}$. 

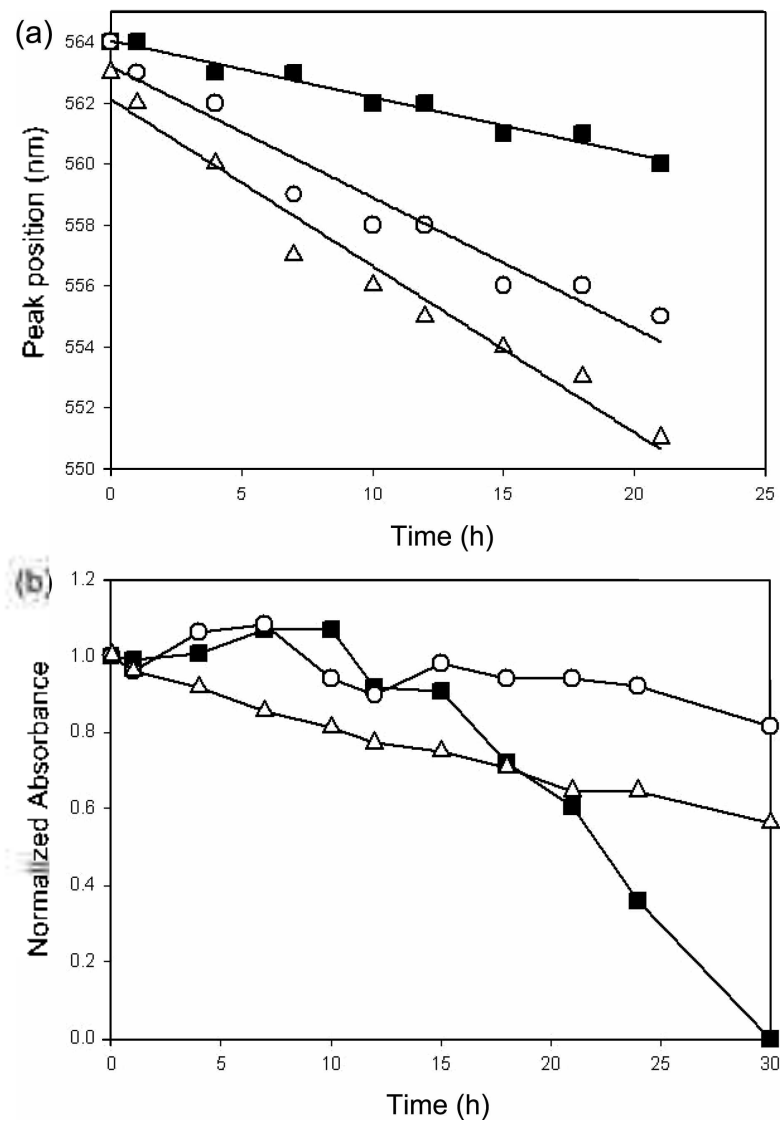

Figure 2. Temporal cvolution of (a) peith positions and (b) peak intensitics of ${ }^{\text {MPA }} \mathrm{QDS}$ (hollow syuar'). ${ }^{\text {GATOPOOD (hollow tircle) }}$ and ${ }^{\mathrm{CTAB}: T O P O} \mathrm{QDS}$ (hollow triangle).

can be seen in Figure 2, "iATCOPO $\mathrm{QD}$ shows superior photochemical stability under the UV exposure condition. In contrast to the decreases in the $I^{\text {st }}$ exciton absorption peak intensities of ${ }^{\mathrm{N} / \mathrm{A} A \mathrm{QD}}$ and ${ }^{(\mathrm{T}+\mathrm{A} / \mathrm{T}) \mathrm{PO}} \mathrm{QD}$. only slight decrease was observed for the absorption peak intensity of (ANTOPOCD. Observed enhancement in photochemical stability of (ANTOPOOD can be explained as follows. As previously suggested by Aldana ef al., ${ }^{4}$ rapid precipitation of MPA QD can be ascribed to the photocatalytic oxidation of thiol groups, which is not applicable for JOPO coated QDS

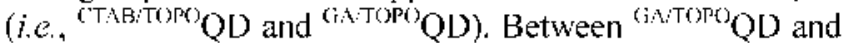
( $T A B \cdot T O P() \mathrm{QD}$, there are two important processes contributing to the differences in photochemical and colloidal stabilities of these QDs: 1) diffusion of oxygen molecules to the QDs surface for photoxidation and 2) transport and collision of QDs to form a coagulated andior precipitated QD. We propose that the extremely complex structural characteristics of GA, highly branched natural polysaccharide with very high molecular weight." are acting as a strong diffusion barrier for both the oxygen molecules and QD nanocrystals and resulted in improved photochemical stability and QY of (IATOPOQD observed in this study.

As a summary, we have demonstrated a simple but effective preparation method of QD nanocolloids in aqueous solution, using biocompatible and non-toxic natural polysaccharides (i.e., Gum Arabic). Moreover, these GA stabili- zed QD nanocolloids have shown significant entancements in their photochemical stabilities as well as quantum yields. which make it as a promising candidate for future applications in biological and medical imaging, such as fluorescence probes for multi-color imaging. Further toxicological experiments using mamıalian cells (e.g., fibroblast) and invertebrate species (e.g. Daphnia magna) to test acute and chronic toxicities of these nanocolloids (i.e.. ${ }^{\mathrm{N} P \mathrm{~A}} \mathrm{QD}$, (TAB:TOPOQD and $\left.{ }^{\circ A T O P O} \mathrm{QD}\right)$ are in progress, which are expected to provide knowledge on the impact of surface modification of nanomaterials on their toxicities.

\section{Experimental Section}

Unless otherwise indicated, all chemicals used in this study were above reagent grade and directly used without further purification. (FAIOPC) $\mathrm{QD}$ nanocolloid was prepared based on the following method, which is previously used by Fan ef al., ${ }^{5} \mathrm{GA}$ was purchased from Sigma-Aldrich and dissolved in deionized water to form 1 wt $\%$ GA solution. Then. GA solution was mixed with well dispersed ${ }^{\mathrm{TOP} O} \mathrm{QD}$ in chloroform $\left(\mathrm{CdSe} / \mathrm{LnSe}\right.$, Core diameter $=3.2 \mathrm{nIn}$, $\lambda_{\mathrm{abs}}=$ $559 \mathrm{~nm}, \lambda_{\mathrm{em}}=577 \mathrm{~nm}$, synthesized using SiPOP methods and generously provided by Nanosquare Inc. for this experiment). The mixed solution was then strongly stirred for 12 I5 hours until the formation of QD microemulsion, which was then slightly heated up to $60^{\circ} \mathrm{C}$ until the complete evaporation of organic solvent. Finally, the QD solution was further filtered with $0.2 \mathrm{~mm}$ syringe filter to remove unwanted large particles.

QDs with different surface modifying molecules such as 3-Mercaptopropionic acid (MPA) and Cetyltrimethylammo-

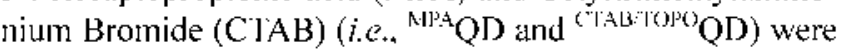
also prepared to compare their colloidal properties with ${ }^{6 A}{ }^{2}()^{\circ} \mathrm{QD}$ nanocolloids. CTAB and MPA were above $99 \%$ purity and purchased from Sigma. For the preparation of ${ }^{M P A} \mathrm{QD}$ nnocolloids in acpueous solution, the TOPO capped QDs were water-solubilized via ligand exchange reaction with MPA, while ${ }^{\text {'TARTOPOOD }}$ was prepared via similar procedure with ${ }^{(\mathrm{A} N \mathrm{~T}) \mathrm{PO}} \mathrm{QD}$ nanocolloids. Further details of surface modification and characterization procedures for these QDs were previously described. ${ }^{16-18}$

Photoluminescence spectra of each QDs and Rhodamine 6G (used as reference compound for $\mathrm{QY}$ measurement, $\mathrm{QY}$ $=0.99$ ) were collected using ISS P Cl Photon Counting Spectrofluorometer (ISS Inc) and Quantum Yields (QY) were calculated based on the IUI'AC method. ${ }^{19}$ The absorption spectra were recorded by using OI'IIZEN $2120 \mathrm{UV} / \mathrm{Vis}$ spectrophotometer (MECASYS Co. Ltd.). Concentrations of QDs in aqueous solutions were determined from the intensities of the first excitonic absorption peak and extinction coefficients based on the method reported by $\mathrm{Yu}$ et $a l^{5}$ and presented in the units of moles of QD per liter (i.e., [QD]). Peak position, height and width of the first exciton absorption of QD nanoparticles were used to monitor the physical changes of $\mathrm{QD}$ particles, i.e; average diameter of QDs. estimated amount of suspended QDs and heterogeneity of 
QD particle size distributions.

All colloidal stability experinents under UV exposure conditions were performed in a photooxidation chamber equipped with a UV lamp (peak at $254 \mathrm{~lm}$ ). During the UV exposure experiments. the QD samples $(-3 \mathrm{~mL})$ contained in $1 \mathrm{~cm}$ PMMA cells, were placed $\sim 18 \mathrm{~cm}$ directly from the UV lanp. The UV/vis absorption spectra were recorded in a certain time interval to monitor changes in QD nanocolloids.

Acknowledgments. This work was supported by Korea Research Foundation Grant funded by the Korean Government (MOEHRD) (KRF-2006-C00607). We also thanks to Nanosquare Co. Ltd. for providing quantum dot raw materials for this study.

\section{References}

1. Murray. C. B.: Norris. D. J: Bawendi. M. G. J. Am. Chem. Soc. 1993, $115.8706-8715$

2. Yu. W. W. Chang. E.: Drezek. R.: Colvin. V. L. Biochem. Bioplys. Res. Commm. 2006. 348.781-786.

3. Dubois. F.: Mahler. B.: Dubertret. B.: Doris. E.: Mioskowski. C. J. Am. Chem. Soc. 2007. 129.482-483.

4. Aldana. J.: Wang. Y. A.: Peng. X. G. J Am. Chem Soc. 2001 . I23. $8844-8850$

5. Fan, H. Y.: Leve. E. W: Scullin. C:: Gabaldon. J: Tallant. D.:
Bunge. S.: Boyle. T.: Wilson. M. C.: Brinker. C. J. Namo Lett. 2005. 5. 645-648.

6. Gao. X. H.: Yang. L. L.: Petros. J. A.: Marshal. F. F.: Simnons. J W.: Nie. S. M. Curent Opinion in Biotecholog, 2005, 16.63-72.

7. Dubertret. B.: Skourides, P.: Norris, D. J.; Noireaux, V.; Brivanlou, A. H., Libchaber, A. Science 2002. 298, 1759-1762.

8. Verbeken. D.: Dierckx. S.: Dewettinck. K. Appl Michobiol. Biotectmol 2003. 63. 10-21

9. Renard. D.: Lavenant-Gourgeon. L.: Ralet. M. C.: Sanchez. C. Bionacromolecules 2006. 7.2637-2649

10. Johnson. W. hit. J. Toxicol. 2005. 2t, 75-118.

11. Bandyopadhyaya, R.: Nativ-Roth. E; Regev, O.: YerushalmiRozent. R. Jano Lett. 2002. 2. 25-28.

12. Kattumuri. V.: Katti. K.: Bhaskaran. S.: Boote. E. J.: Casteel. S. W: Fent. G. M.: Robertsont. D. J.: Chandrasekhar. M.: Kant1an. R. Katti. K. V. Small 2007.3, 333-341.

13. Velikov, K. P. Zegers. G. E.: van Blaaderen. A. Lamgmiir 2003. 19. 1384-1389.

14. Williams. D. N.: Gold. K. A.: Holomant. T. R. P.: Elrmant. S. H.: Wilsont. O. C. J. Konopart. Res. 2006. 8. 749-753.

15. Zhang. H. Y.: Shan. G. B.: Liu. H. Z.: Xing. I. M. Sinf. Coat. Techol. 2007. 201,6917-6921

16. Morshed, A. M. A.; Yoon, T. H. Bull. Kowam Chem. Soc. 2008. 29.249-251

17. Park. C.: Kinn. D. H.: Kim. M. J.: Yoon. T. H. Bull Korean Chent Soc. $2008.29 .303-304$

18. Kimn. M. T.: Park. C.: Choi. K.: Yoon. T. H. Bull Korean Chent. Soc. 2008, 29, 1101-1102

19. Eaton, D. ILPAC. 1998. 60, 1107-1114. 